\title{
POLARIZED XANES OF IRON PORPHYRINS
}

\author{
S. Wang, G.S. Waldo, R. Fronko, and J.E. Penner-Hahn \\ Dept. of Chemistry, University of Michigan, Ann Arbor
}

XANES is known to be very sensitive to the structural and electronic details of the absorbing site. In particular, several workers have noted the potential of XANES for elucidating the local three-dimensional structure of the iron sites in heme proteins $[1,2]$. In order to clarify the specific correlation between spectral features and molecular structure, we have measured polarized $\mathrm{X}$-ray absorption spectra for a series of Fe porphyrins.

An expansion of the $1 s \rightarrow 3 d$ region for $\left.F e(T P P) F_{2}\right]^{-}$is shown in Figure 1. The isotropic spectrum shows a broad peak with some evidence that multiple unresolved transitions contribute to this peak. In contrast, the two in-plane spectra show relatively narrow peaks centered at energies which depend on orientation. In addition, there is a slight difference in energy between the peaks of the in-plane (e) $F e-N$ ) and the axial orientations.

For the tetragonally compressed Fe porphyrin, the $d$ orbital energies are: $d_{x y}<$ $d_{x z, y z}<d_{z} 2<d_{x} 2 y^{2}$. For a centrosymmetric complex, the transitions are governed predominantly by quadruple selection rules $[3]$. When $\mathbf{e}$ is in the axial orientation, only the $1 s \rightarrow d_{x z, y z}$ transitions will be observed. The $1 s \rightarrow d_{x y}$, $1 s \rightarrow d_{x} 2_{-y} 2.1 s \rightarrow d_{x z, y z}$ transitions will be allowed, with the relative intensities of the $1 s \rightarrow d_{x y}$ and $1 s \rightarrow d_{x} 2_{-y} 2$ transitions depending on the angle between $e$ and the $\mathrm{Fe}-\mathrm{N}$ bonds. Our results for high spin $\left[F e(T P P) F_{2}\right]^{-}$are consistent with this model. A low energy transition $(7112.6 \mathrm{eV})$ is observed when $\mathbf{e}$ is parallel to one of the $\mathrm{Fe}-\mathrm{N}$ bonds. We assign this transition as predominantly $1 s \rightarrow d_{x y}$. For the orientation in which e bisects two Fe-N bonds, a higher energy transition is observed at $7114.5 \mathrm{eV}$ (assigned as predominantly $1 s \rightarrow d_{x}{ }^{2} y^{2}$ ) is observed. The axial spectrum, with a peak at $c a .7113 \mathrm{eV}$, is consistent with the expected transitions $1 s-d_{x z, y z}$. These data illustrate the potential of polarized measurements for simplifying the interpretation of XANES spectra.

Figure 2a shows the in-plane polarized spectra of several 6 coordinate $F E$ (III) porphyrins, and the Fig. 2b shows the corresponding axial spectra. The in-plane spectra for the 6 coordinate porphyrins are very similar to each other. This is reasonable since the structural variations in the porphyrin moiety are relatively minor. In contrast, there are significant differences between axial and in-plane spectra. For example, the intra-compound variation can be as large as $3-4 \mathrm{eV}$. This suggests that, at least for these systems, bond length plays a major role in determining the absorption edge energy. Similar conclusions were reached by Bianconi et al. on the basis of theoretical calculations [4].

A careful examination of the in-plane spectra shows that the relative heights of the peaks in the 7160-7210 eV range vary from compound to compound. Given the polarization, it is reasonable to conclude that these changes are due to scattering from the porphyrin plane. A reasonable explanation for this result is that as the 

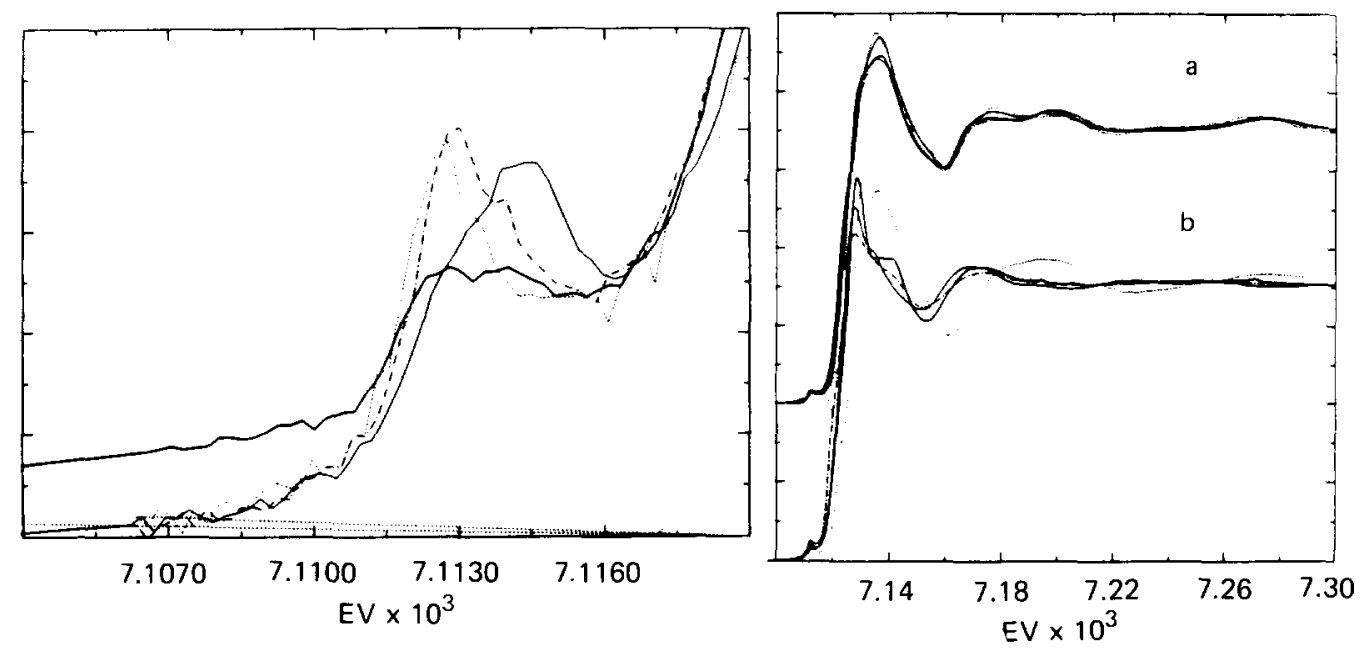

Fig.1 (left): XANES spectra for $\left|F e(T P P) F_{2}\right|$-. Dark: Isotropic; Dat-dash: e|z; Dotted, e $F e-N$; Light: e in $F e N_{\mathbb{4}}$ plane, bisecting $F e-N$ bonds.

Fig. 2 (right): Polarized spectra for 6-coordinate $\mathrm{Fe}(\mathrm{III})$ porphyrins. Dark: $\mid \mathrm{Fe}(O E P)(3-$ $\left.C l P_{y}\right)_{2} ! C l O_{4}$ : Dot-Dash: $\left|\mathrm{Fe}(\mathrm{OEP})(\mathrm{THF})_{2}\right| \mathrm{ClO}_{4}$; Light solid: $\left[\mathrm{Fe}(\mathrm{OEP})\left(2-\mathrm{MeIm}_{2} \mid \mathrm{Clo}_{4}\right.\right.$; Dotted $\mathrm{Fe}(\mathrm{TPP}) \mathrm{F}_{2} \mathrm{ClO}_{4}$. A) In-plane polarized spectra. B) Axially polarized spectra.

Fe- $\mathrm{N}_{4}$ core expands, the interference between the low frequency $\mathrm{N}$ scattering and higher frequency $\mathrm{C}$ scattering changes. A generally similar conclusion was drawn by Chance et al., who referred to these transitions as the "Ligand Field Indicator Region" (LFIR) |5. An important advance of the current study is direct experimental determination that these transitions arise predominantly from scattering within the prophyrin plane. Since these porphyrins were chosen to have the Fe in the center of the porphyrin plane, it is clear that these changes are not caused by the Fe out-of-plane displacement. It has been suggested that these transitions can be used as general state-spin markers. Our data demonstrate that, while this will be true for selected high-spin/low spin comparisons, caution must be exercised in interpreting powder XANES spectra. Comparison of Figs. 2a,b shows that the superposition of axial and equatorial contributions can significantly change the ratio of the "LFIR" peaks.

In summary, we have demonstrated that polarized measurements are an important technique for understanding Fe porphyrin XANES spectra.

Acknowledgements. This work was partly supported by the donors of the Petroleum Research Foundation (18073-G3,6). JEPH is the recipient of a Camille and Henry Dreyfus Distinguished Young Faculty Award.

1. M.R. Chance. et al., J. Bio. Chem. 261 (1986) 5689-5692.

2. A. Bianconi. et al. Nature 318 (1985) 685-687.

3. J.E. Hahn, et al. Chem. Phys. Lett. 88 (1982) 595-598.

4. A. Bianconi et al., Biophys. Res. Comm. 131 (1985) 98-102.

5. B. Chance, et al., Arch. Biochem. Biophys. 235 (1984) 596-611. 